\title{
Prediction of Mortality by Hematological Parameters on Admission in Patients With Subarachnoid Hemorrhage
}

\author{
Nobutake SADAmASA, ${ }^{1}$ Kazumichi YOSHIDA, ${ }^{1}$ Osamu NARUMI, ${ }^{1}$ \\ Masaki CHIN, ${ }^{1}$ and Sen YAMAGATA ${ }^{1}$ \\ ${ }^{1}$ Department of Neurosurgery, Kurashiki Central Hospital, Kurashiki, Okayama
}

\begin{abstract}
This retrospective study investigated the correlations between red blood cell (RBC) count, hemoglobin, and hematocrit on admission, and mortality in 140 patients with subarachnoid hemorrhage (SAH). Correlations between the hematological parameters RBC count, hematocrit, hemoglobin, white blood cell (WBC) count, and platelet count on admission and mortality were investigated. Association of each variable with mortality was tested by univariate and multivariate logistic regression analysis. Univariate analysis showed that mortality was associated with RBC count, hematocrit, hemoglobin, and platelet count. Multivariate logistic regression analysis revealed hematocrit, WBC count, and platelet count were significant independent predictors for mortality after SAH. Hematocrit was a new significant predictor related to mortality in patients with SAH.
\end{abstract}

Key words: subarachnoid hemorrhage, mortality, hematocrit, white blood cell, platelet

\section{Introduction}

Subarachnoid hemorrhage (SAH) remains a lifethreatening condition, but grading on admission using the Hunt and Kosnik and World Federation of Neurological Societies (WFNS) scales can indicate the prognosis. Several biological markers including d-dimer ${ }^{1)}$ and glial fibrillary acidic protein ${ }^{7)}$ have the potential to predict the outcome on admission, but the most simple and longest used method is white blood cell (WBC) count, which was first reported to correlate with the prognosis in patients with SAH in 1974. ${ }^{5}$ The trend in WBC count and platelet count are also reported to be predictors of vasospasm, ${ }^{2,6}$ which is thought to be one of the significant determinants of outcome after SAH. However, no study has examined the correlation with other hematological parameters, such as red blood cell (RBC) count, hemoglobin, and hematocrit on admission, and mortality in patients with SAH.

This retrospective study investigated the relationship between hematological parameters on admission and mortality in patients with SAH.

\section{Materials and Methods}

The clinical records of patients with acute nontrau-

Received April 5, 2011; Accepted June 29, 2011

matic SAH who were admitted to Kurashiki Central Hospital between January 2005 and October 2006 were retrospectively reviewed. SAH was confirmed in all patients by head computed tomography (CT) and was rated according to the Fisher scale. Subsequently, head three-dimensional CT angiography was performed to detect bleeding sources such as intracranial aneurysm. Patients in severe condition that did not allow use of CT angiography or other angiographic modalities were classified as "not identified." WFNS grade and hematological parameters from complete blood count such as RBC count, hematocrit, hemoglobin, WBC count, and platelet count on admission before any surgical or medical treatment was recorded. Unknown SAH was defined as no bleeding source detected by CT angiography or other angiographical modalities. Patients with normal pressure hydrocephalus that needed ventriculoperitoneal shunting were classified as "shunt needed." Delayed ischemic neurological deficit (DIND) was defined as worsening of the neurological condition (altered consciousness, aphasia, and hemiplegia or hemiparesis) that could not be attributed to rebleeding, postoperative complications, hydrocephalus, or systemic complications. Outcome was assessed at 3 months after SAH according to the modified Rankin scale (mRS). A complete record of daily examinations, surgical procedures, and clinical course was maintained for each 
patient.

Data are presented as mean \pm standard deviation for continuous variables, and as frequency for categorical variables. Association of continuous variables and/or gradings was tested by the Spearman correlation coefficient. The unpaired t-test was used to compare for the hematological parameters and age, and the chi-square test was used for WFNS grade and Fisher group in univariate analyses. Multivariate logistic regression analyses were performed to compare the two groups (mRS 0-5 and mRS 6), using variables showing $\mathrm{p}<0.1$ on the univariate analyses. The cut-off points of each continuous variable for predicting mortality were obtained using receiver operating characteristic curves. A p value less than 0.05 was considered significant for all analyses.

\section{Results}

The baseline characteristics of 140 patients with SAH on admission are shown in Table 1. Mean hematological parameters were within the standard ranges except for WBC count $\left(10.7 \pm 4.5 \times 10^{3} / \mu \mathrm{l}\right)$, which was higher than the upper limit of standard range in our institution. All patients with SAH of unknown origin survived, and the four patients who died were classified as "not identified." Age was significantly higher in the patients who died than in the survivors $(70.0 \pm 13.3$ vs. $62.8 \pm 13.6$ years, $p=$ 0.018 , unpaired t-test). The patients with mortality were significantly more likely to be classified as WFNS grade 5, Fisher group 4, and no surgical treatment, and less likely to be classified as "shunt needed" than the survivors. Sex, multiple aneurysms, length of hospital stay, and DIND were not significantly associated with mortality (Table 1).

WFNS grade was significantly correlated with WBC count ( $\mathrm{r}=0.369, \mathrm{p}<0.0001)$, but not with RBC count, platelet count, and age by Spearman correlation coefficients. Patient age was significantly correlated with RBC count $(\mathrm{r}=-0.443, \mathrm{p}<$ 0.0001), hematocrit $(\mathrm{r}=-0.332, \mathrm{p}<0.0001)$, hemoglobin $(\mathrm{r}=-0.332, \mathrm{p}<0.0001)$, and platelet count ( $r=-0.231, p=0.0065)$, but not with WBC count and Fisher group. Fisher group was significantly correlated with WBC count $(\mathrm{r}=0.357, \mathrm{p}$ $<0.0001$ ), hematocrit ( $\mathrm{r}=-0.331, \mathrm{p}<0.0001)$, and hemoglobin $(\mathrm{r}=-0.332, \mathrm{p}<0.0001)$, whereas did not correlate with RBC count.

Univariate analysis showed that patients who died (mRS 6) had significantly lower RBC count (4.26 \pm 0.57 vs. $\left.3.82 \pm 0.69 \times 10^{6} / \mu \mathrm{l}, \quad \mathrm{p}=0.001\right)$, lower hematocrit $(39.7 \pm 4.97 \%$ vs. $36.4 \pm 6.01 \%, \quad p=$ 0.005), lower hemoglobin (13.2 \pm 1.83 vs. $12.2 \pm$
Table 1 Baseline characteristics of 140 patients with subarachnoid hemorrhage

\begin{tabular}{|c|c|c|c|}
\hline & mRS $0-5$ & mRS 6 & $\mathrm{p}$ Value \\
\hline No. of patients & 116 & 24 & \\
\hline Sex & & & $0.63^{*}$ \\
\hline male & 36 & 9 & \\
\hline female & 80 & 15 & \\
\hline Location of aneurysm & & & \\
\hline anterior circulation & 88 & 16 & $0.75^{*}$ \\
\hline posterior circulation & 19 & 4 & \\
\hline unknown & 9 & 0 & $0.36^{*}$ \\
\hline not identified & 0 & 4 & $0.007^{*}$ \\
\hline Multiple aneurysms & 9 & 3 & $0.43^{*}$ \\
\hline Age (mean \pm SD), years & $62.8 \pm 13.6$ & $70 \pm 13.3$ & $0.018^{\dagger}$ \\
\hline $\begin{array}{l}\text { Length of stay (median), } \\
\text { days }\end{array}$ & 34 & 3 & $0.16^{\dagger \dagger}$ \\
\hline WFNS grade & & & $<0.0001^{* *}$ \\
\hline 1 & 57 & 2 & \\
\hline 2 & 6 & 1 & \\
\hline 3 & 7 & 0 & \\
\hline 4 & 26 & 2 & \\
\hline 5 & 20 & 19 & \\
\hline Fisher group & & & $0.0002^{* *}$ \\
\hline 1 & 6 & 0 & \\
\hline 2 & 25 & 1 & \\
\hline 3 & 70 & 11 & \\
\hline 4 & 15 & 12 & \\
\hline Surgery & & & $<0.0001^{* *}$ \\
\hline clipping & 79 & 5 & \\
\hline coil embolization & 21 & 2 & \\
\hline other & 1 & 2 & \\
\hline none & 15 & 15 & \\
\hline Shunt needed & 24 & 0 & $0.0014^{*}$ \\
\hline DIND & 17 & 2 & $0.53^{*}$ \\
\hline
\end{tabular}

${ }^{*}$ Fisher exact test, ${ }^{* *}$ chi-square test, ${ }^{\dagger}$ unpaired t-test, ${ }^{\dagger}$ Mann-Whitney U test. DIND: delayed ischemic neurological deficit, mRS: modified Rankin scale, SD: standard deviation, WFNS: World Federation of Neurological Societies.

Table 2 Univariate analysis of the hematological parameters in 140 patients with subarachnoid hemorrhage

\begin{tabular}{lccc}
\hline & $\begin{array}{c}\text { mRS 0-5 } \\
(\mathrm{n}=116)\end{array}$ & $\begin{array}{c}\text { mRS 6 } \\
(\mathrm{n}=24)\end{array}$ & p Value $^{\dagger}$ \\
\hline RBC count, $\times 10^{6} / \mu \mathrm{l}$ & $4.26 \pm 0.57$ & $3.82 \pm 0.69$ & 0.001 \\
MCV, fl & $93.6 \pm 6.6$ & $95.9 \pm 7.9$ & 0.136 \\
MCH, pg & $31.0 \pm 2.7$ & $31.8 \pm 3.2$ & 0.179 \\
MCHC, g/dl & $33.1 \pm 1.2$ & $33.1 \pm 2.0$ & 0.802 \\
Hematocrit, \% & $39.7 \pm 5.0$ & $36.4 \pm 6.0$ & 0.005 \\
Hemoglobin, g/dl & $13.2 \pm 1.8$ & $12.1 \pm 2.1$ & 0.011 \\
WBC count, $\times 10^{3} / \mu \mathrm{l}$ & $10.3 \pm 3.8$ & $12.2 \pm 6.8$ & 0.070 \\
Platelet count, $\times 10^{4} / \mu \mathrm{l}$ & $21.6 \pm 6.5$ & $18.5 \pm 8.0$ & 0.043 \\
\hline
\end{tabular}

Data are expressed in mean \pm standard deviation. 'Unpaired t-test. MCH: mean corpuscular hemoglobin, MCHC: mean corpuscular hemoconcentration, MCV: mean corpuscular volume, mRS: modified Rankin scale, RBC: red blood cell, WBC: white blood cell. 
Table 3 Multivariate logistic regression analysis of the hematological parameters, age, and Fisher group for mortality (mRS 6) after subarachnoid hemorrhage

\begin{tabular}{lcll}
\hline & Odds ratio & \multicolumn{1}{c}{$95 \%$ CI } & p Value \\
\hline RBC count $<4.6 \times 10^{6} / \mathrm{ml}$ & 0.332 & $0.063-1.756$ & 0.1948 \\
Hematocrit $<38.5 \%$ & 8.072 & $1.076-60.514$ & 0.0421 \\
Hemoglobin $<12.7 \mathrm{~g} / \mathrm{dl}$ & 0.700 & $0.113-4.344$ & 0.7019 \\
WBC count $>10.5 \times 10^{3} / \mathrm{ml}$ & 2.434 & $0.826-7.163$ & 0.1062 \\
Platelet count $<20 \times 10^{4} / \mathrm{ml}$ & 3.399 & $1.088-10.610$ & 0.0351 \\
Age $>67$ years & 2.632 & $0.845-8.205$ & 0.0954 \\
Fisher group 3 or 4 & 6.200 & $0.733-52.376$ & 0.0940 \\
\hline
\end{tabular}

CI: confidence interval, mRS: modified Rankin scale, RBC: red blood cell, WBC: white blood cell.

$2.11 \mathrm{~g} / \mathrm{dl}, \mathrm{p}=0.011)$, and lower platelet count $(21.6$ \pm 6.51 vs. $\left.18.5 \pm 7.95 \times 10^{4} / \mu \mathrm{l}, \mathrm{p}=0.043\right)$ than the patients with mRS 0-5 (Table 2). Multivariate logistic regression analysis of the hematological parameters showed hematocrit, WBC count, and platelet count were significantly associated with mortality. Hematocrit was also correlated with age and Fisher group, so we included age and Fisher group in the multiple logistic regression analysis, and found that hematocrit and platelet were significantly associated with mortality (Table 3).

\section{Discussion}

The present study found that RBC count, hematocrit, and hemoglobin on admission were correlated with mortality after SAH. Univariate analysis showed that RBC count, hemoglobin, and hematocrit were associated with mortality after $\mathrm{SAH}$, whereas multiple logistic regression analysis showed that only hematocrit was an independent predictor for mortality after SAH. Why low RBC count, low hemoglobin, and low hematocrit were correlated with mortality after SAH remains unclear. RBC mass and total blood volume were significantly decreased in the acute phase of $\mathrm{SAH}$ patients, ${ }^{3)}$ indicating hypovolemia in the acute phase of SAH. Therefore, hypervolemia is one of the obvious treatment options to prevent ischemic complication derived from symptomatic vasospasm. The amount of cerebral blood flow in the acute phase is correlated with the clinical grade of SAH. ${ }^{9)}$ Apparently, poor clinical grade is linked to poor outcome. Consequently, cerebral ischemia in the acute phase of SAH is a significant determinant of poor outcome. Low RBC count, low hemoglobin, and low hematocrit on admission might be the result of acute hypovolemia, and so might decrease the tolerance against cerebral ischemia in the acute (and possibly chronic) phase of SAH.
Low RBC count, low hemoglobin, and low hematocrit might result from the substantial blood loss caused by the SAH. We could not compare these parameters before and after the onset of SAH. The only indicator of hematoma volume measured in this study was Fisher scale. Leucocytosis in the acute phase of SAH is considered as an acute inflammatory response which is caused by subarachnoid blood, and seems to be correlated with hematoma volume (Fisher scale), ${ }^{11)}$ although the correlation between Fisher scale and RBC count was not examined. In our study, Fisher scale was not correlated with RBC count, but was correlated with hemoglobin, hematocrit, and WBC count. These findings indicate that decreases in those RBC-related parameters in the patients who died were not fully explained by the hematoma volume indicated by Fisher group. On the other hand, age was inversely correlated with RBC count, hematocrit, hemoglobin, and platelet count in this study, suggesting that these parameters might reflect age instead of WFNS grade. We did show that hematocrit and platelet are independent risk factors for poor prognosis based on multiple logistic regression analysis of the hematological parameters together with age and Fisher group. Both age and WFNS grade were previously reported as predictors for poor outcome after SAH. ${ }^{8)}$ Our Spearman correlation coefficient results also showed that age and WFNS grade were significantly associated with poor prognosis. Our study supports the importance of these factors as predictors for outcome after SAH.

In contrast to hematocrit, our study found no correlation between hemoglobin and mortality by multivariate analysis. Higher hemoglobin levels on admission are associated with less cerebral infarction and improved outcome after $\mathrm{SAH},{ }^{4}$ although no rationale was advanced. On the other hand, lower hemoglobin (and apparently lower hematocrit) reduces blood viscosity and enhances blood flow, ${ }^{10)}$ so that hemodilution therapy is performed in general for the treatment of symptomatic vasospasm. Hemoglobin should be raised on admission to improve the prognosis, but this study does not indicate the optimum hemoglobin level on admission for best outcome after SAH.

Hematocrit is a new significant predictor for mortality in patients with SAH. Hematocrit and platelet were correlated with age, and WBC count with WFNS grade. Low hematocrit might be an indicator for hypovolemia in the acute phase of SAH. Further investigation is needed to clarify the relationship between hematological parameters and SAH. 


\section{Disclosure}

No funding source contributed to this research.

\section{References}

1) Juvela S, Siironen J: D-dimer as an independent predictor for poor outcome after aneurysmal subarachnoid hemorrhage. Stroke 37: 1451-1456, 2006

2) Kasius KM, Frijns CJM, Algra A, Rinkel GJE: Association of platelet and leukocyte counts with delayed cerebral ischemia in aneurysmal subarachnoid hemorrhage. Cerebrovasc Dis 29: 576-583, 2010

3) Maroon JC, Nelson PB: Hypovolemia in patients with subarachnoid hemorrhage: therapeutic implications. Neurosurgery 4: 223-226, 1979

4) Naidech AM, Jovanovic B, Wartenberg KE, Parra A, Ostapkovich N, Connolly ES, Mayer SA, Commichau $\mathrm{C}$ : Higher hemoglobin is associated with improved outcome after subarachnoid hemorrhage. Crit Care Med 35: 2383-2389, 2007

5) Neil-Dwyer G, Cruickshank J: The blood leucocyte count and its prognostic significance in subarachnoid haemorrhage. Brain 97: 79-86, 1974

6) Niikawa S, Hara S, Ohe N, Miwa Y, Ohkuma A: Correlation between blood parameters and symptomatic vasospasm in subarachnoid hemorrhage patients. Neurol Med Chir (Tokyo) 37: 881-884, 1997
7) Nylén K, Csajbok LZ, Ost M, Rashid A, Blennow K, Nellgård B, Rosengren L: Serum glial fibrillary acidic protein is related to focal brain injury and outcome after aneurysmal subarachnoid hemorrhage. Stroke 38: 1489-1494, 2007

8) Rosengart AJ, Schultheiss KE, Tolentino J, Macdonald RL: Prognostic factors for outcome in patients with aneurysmal subarachnoid hemorrhage. Stroke 38: 2315-2321, 2007

9) Solomon RA, Post KD, McMurtry JG 3rd: Depression of circulating blood volume in patients after subarachnoid hemorrhage: implications for the management of symptomatic vasospasm. Neurosurgery 15: 354-361, 1984

10) Thomas DJ, Marshall J, Russell RW, Wetherley-Mein G, du Boulay GH, Pearson TC, Symon L, Zilkha E: Effect of haematocrit on cerebral blood-flow in man. Lancet 2: 941-943, 1977

11) Yoshimoto Y, Tanaka Y, Hoya K: Acute systemic inflammatory response syndrome in subarachnoid hemorrhage. Stroke 32: 1989-1993, 2001

Address reprint requests to: Nobutake Sadamasa, MD, PhD, Department of Neurosurgery, Kurashiki Central Hospital, 1-1-1 Miwa, Kurashiki, Okayama 710-8602, Japan. e-mail: ns11267@kchnet.or.jp 